\title{
Adaptação transcultural do questionário HIV/AIDS-Target Quality of Life para avaliação da qualidade de vida em pacientes com HIV/aids
}

\author{
Transcultural adaptation of the HIV/AIDS-Target Quality of Life \\ for HIV/AIDS for the evaluation of patients' quality of life
}

Ana $M$ aria $M$ artins $M$ oser ${ }^{~}$

Jefferson Traebert ${ }^{2}$

${ }^{1}$ Universidade do O estede Santa Catarina. Rua Oiapoc 211, Bairro Agostini. 89900-000 São Miguel do Oeste SC.

anamoser@saude.sc.gov.br ${ }^{2}$ Universidade do Sul de Santa Catarina.
Abstract The objectives of this study were to carry out cross-cultural adaptation of theH IV/AIDSTarget Quality of Life Instrument for Portuguese and to observe its psychometric properties. Translations, translations synthesis, back translation, analysis by a committee of specialists, pre-final version test, studies of validity and reliability of the final version were carried out. Psychometric properties were evaluated by the administration of the Brazilian version in 50 consecutive HIV positive patients, from the DST/HIV/AIDS municipal ambulatory of São M iguel do O este, Santa Catarina State. The internal consistency was observed by $\alpha$-Cronbach. The reliability was estimated using the intraclass correlation coefficient and Bland-Altmann diagram. The validity was observed comparing the scores of thequestionnaire with theW HOQ OL-HIV-Brief through the Spearman rank correlation coefficient. Theresults pointed out to $\alpha$-Cronbach of 0.893 . The correlations obtained were 0.934 for inter-interviewer and 0.977 for intra-interviewer. The correlation with WHOQ OL-HIV-Bref was 0.824. It was concluded that the cross-cultural adaptation process was successful and the adapted questionnaire demonstrated good psychometric properties.

Key words Questionnaires, Cross-cultural comparison, Q uality of life, Acquired immunodeficiency syndrome
Resumo 0 objetivo desse estudo foi realizar a adaptação transcultural do questionário HIV/ AIDS-Target Quality of Life Instrument para 0 português e avaliar suas propriedades psicomé tricas. Foram realizadastraduções, síntese dastraduções, retrotradução, análise por comitê de especialistas, teste da versão préfinal, estudos de validade e confiabilidade. As propriedades psicométricas foram avaliadas pela administração da versão brasileira em cinquenta pacientes consecutivos soropositivos atendidos no Ambulatório de DST/HIV/aids de São M iguel do O este (SC). O bservou-se a consistência interna através do $\alpha$ Cronbach. A confiabilidade foi estimada pelo coeficiente de correlação intraclasses e diagrama de Bland-Altmann. A validade foi observada comparando os escores do instrumento com o W HOQ OL-HIV-Bref por meio do coeficiente de correlação por postos deSpearman. O sresultados apontaram para um $\alpha$-Cronbach de 0,893. A correlação inter-entrevistador foi de 0,934 e intra-entrevistador de 0,977. A correlação com WHOQ OL-HIV-Bref foi de 0,824 . Pôde seconcluir que o processo de adaptação transcultural obteve sucesso e o instrumento adaptado demonstrou boas propriedades psicométricas.

Palavras-chave Questionários, Comparação transcultural, Qualidade de vida, Síndrome de imunodeficiência adquirida 


\section{Introdução}

Nos últimos anos, registraram-se grandes progressos na prevenção e no controle do HIV/aids. Destaque especial aos antirretrovirais, os quais, desde 1996, reduziram em até $90 \%$ a mortalidadenos países industrializados ${ }^{1}$. Dentro destecontexto, novas questões parecem ganhar importância, pois anteriormentepriorizava-se a sobrevivência, existindo agora a preocupação com a qualidade de vida dos infectados².

A Organização M undial de Saúde, em estudo multicêntrico, com o objetivo elaborar um instrumento que avaliasse qualidade de vida (QV) em uma perspectiva internacional e transcultural, adotou o seguinte conceito de QV: “a percepção do indivíduo acerca de sua posição na vida, de acordo com o contexto cultural e os sistemas de valores nos quais vive, e em relação a seus objetivos, expectativas, padrões e preocupações" ${ }^{3}$. Este conceito engloba seis diferentes domínios: saúde física, estado psicológico, níveis de dependência, relacionamento social, características ambientais e padrão espiritual. 0 grupo quetrabalhou este conceito concluiu que a avaliação em diferentes domínios permitea verificação das dimensões nas quais os tratamentos serão efetivos, podendo direcionar medidas terapêuticas mais adequadas e provavelmente diminuir os custos em saúde 3 . I sto espelha a natureza subjetiva da avaliação, ou seja, é a percepção do respondente/paciente que deve ser avaliada ${ }^{4}$.

O sinstrumentos queavaliam QV classificamse em genéricos eespecíficos. Os primeiros avaliam de forma global os aspectos relacionados à qualidade de vida, sejam eles físico, mental, psicológico, emocional, sexual, entre outros. Os específicos concentram-se na avaliação de forma específica de alguns aspectos da QV relacionados subsequentementeà experiência de doenças, agravos ou intervenções médicas 5 .

Dentre os questionários específicos para portadores do HIV/aids, destacam-se o HIV/AIDSTarget Quality of Life (HAT-QOL) ${ }^{6}$ e o WHOQOL-HIV-Bref ${ }^{7,8}$.

O HAT-QOL busca avaliar a QV sob nove domínios: funções gerais, funções sexuais, problemas de comunicação, preocupações com a saúde, preocupações financeiras, aceitação sobre o HIV, satisfação com a vida, preocupações com medicamentos e confiança no serviço de saúde, em um total de 42 questões. As respostas são configuradas em uma escala do tipo Likert com cinco opções, variando de 1 (todo o tempo) a 5 (nenhuma parte do tempo). Os escores são calculados e convertidos em índices de ponderação que variam de 0 a 1006. Já o WHOQOL-HIVBref é composto por cinco questões HIV-específicas para pessoas infectadas, além daquelas que compõem o WHOQOL-Bref. Está dividido em seisdomínios: físico, psicológico, nível de dependência, relação social, meio ambiente eespiritualidade/religião ecrenças pessoais, distribuídos em 31 questoes ${ }^{7,8}$.

0 objetivo desse trabalho foi traduzir para o português, realizar a adaptação transcultural para a cultura brasileira e validar o instrumento HIV/ AIDS-Target Quality of Life, avaliando suas propriedades psicométricas.

\section{Métodos}

Trata-se de um estudo transversal para validação de questionário. Foram entrevistados sessenta pacientes consecutivos, soropositivos doentes ou não, que frequentaram o Ambulatório deDST/HIV/aids da Secretaria M unicipal da Saúde do município de São Miguel do Oeste (SC), no período de abril a julho de 2006. Destes, dez foram entrevistados na etapa do pré teste e cinquenta foram entrevistados na etapa de avaliação das propriedades psicométricas. Os critérios de exclusão foram os seguintes: pacientes com estado clínico que impedisse a real ização das entrevistas e pacientes analfabetos.

0 projeto de pesquisa foi aprovado pelo Comitê de Ética em Pesquisa da Universidade do Oeste de Santa Catarina e todos os pacientes assinaram o termo de consentimento livre e esclarecido, redigido deacordo com os padrões exigidos pela Declaração de Helsinki. Salienta-se a obtenção, por escrito, por parte dos autores do instrumento original, de autorização para proceder a tradução e adaptação transcultural do H AT-QOL para o idioma português, no contexto da cultura brasileira.

\section{Processo deadaptação cultural}

As diretrizes seguidas nessa pesqui sa foram propostas por Beaton et al. ${ }^{9} \mathrm{eW}$ ild et al. ${ }^{10}$. Os passos são descritos a seguir.

Tradução inicial para o idioma Português falado no Brasil

A tradução direta do questionário H AT-QO L em sua versão original do idioma inglês para o idioma português falado no Brasil foi realizada por um tradutor juramentado, sem conhecimen- 
to médico, vínculo acadêmico, não sabedor do propósito do estudo e por profissional da saúde da área da enfermagem, com vínculo acadêmico e sabedor do propósito do estudo.

\section{Síntese das traduções}

As duas traduções foram comparadas e sintetizadas em uma única versão brasileira pelos autores da pesquisa e por dois professores do idioma inglês, não nativos.

\section{Retrotradução}

A retrotradução foi realizada por dois professores de inglês, nativos, sem qualquer conhecimento médico e do questionário original.

\section{Comitê de especialistas}

O comitê de especialistas foi composto pelos profissionais envolvidos na pesquisa, duas professoras do idioma inglês e uma professora doutora em língua inglesa. Esse comitê avaliou as equivalências semântica, idiomática, experimental econceitual. A pós identificar e discutir discrepâncias, foi elaborada versão pré-final.

Teste da versão pré-final

0 questionário em sua versão pré-final foi administrado em dez pacientes infectados pelo HIV e/ou doentes de aids.

\section{Análise dos resultados do pré-teste}

Os pesquisadores analisaram os resultados do préteste e, juntamente com uma professora doutora em língua portuguesa, aprovaram a versão pré-final como versão final.

\section{Propriedades psicométricas}

A análise da consistência interna dos domínios do HAT-QOL foi realizada por intermédio do coeficiente $\alpha$-Cronbach padronizado.

A reprodutibilidade intra-entrevistador foi testada mediante a aplicação de duas entrevistas, realizadas por um único entrevistador, junto aos cinquenta pacientes. 0 intervalo entre as duas entrevistas variou entre quatro e cinco dias. Para avaliação da reprodutibilidade inter-entrevistador, uma terceira entrevista foi realizada por um médico no mesmo dia da primeira entrevista. Tanto a reprodutibilidade intra como inter-entrevistador foi analisada por intermédio do coe- ficiente de correlação intraclasse (ICC $)^{11}$ e pelo método de Bland e Altman ${ }^{12}$.

Para análise da validade, compararam-se os resultados da primeira entrevista do HAT-QOL com os resultados da aplicação do WHOQ OLHIV-Bref. Os resultados foram analisados mediante o coeficiente de correlação por postos de Spearman ${ }^{13}$.

\section{Resultados}

A média de idade dos pacientes foi de 37 anos ( $D P=10,1)$ e $54,0 \%$ eram do sexo feminino. A Tabela 1 mostra outras informações sobreo perfil dos pacientes estudados.

Em relação à analise da consistência interna, a versão brasileira do HAT-QOL mostrou um valor $\alpha$-Cronbach de 0,893 . Ainda foi calculado o valor $\alpha$-Cronbach caso cada um dos domínios fossem excluídos do HAT-QOL, uma vez que o valor dessa apuração estatística tem o intuito de representar numericamente a uniformidade ou tendência nas respostas dentro de cada domínio do questionário. Os valores estão apresentados na Tabela 2.

0 ICC intra-entrevistador foi de 0,977 (IC $95 \% 0,958-0,988)(p<0,001)$. Já a reprodutibili-

Tabela 1. Perfil dos pacientes estudados. São Miguel do O este (SC), 2006. $(n=50)$.

\begin{tabular}{lrr}
\hline & $\mathrm{n}$ & $\%$ \\
\hline Sexo & & \\
M asculino & 23 & 46,0 \\
$\quad$ Feminino & 27 & 54,0 \\
Condição marital & & \\
$\quad$ Com companheiro(a) & 27 & 54,0 \\
$\quad$ Sem companheiro(a) & 23 & 46,0 \\
Grau de instrução & & \\
$\quad$ Fundamental & 14 & 28,0 \\
$\quad$ M édio & 31 & 62,0 \\
Autoavaliação do estado de saúde & & \\
$\quad$ Muito boa & 11 & 22,0 \\
$\quad$ Boa & 20 & 40,0 \\
$\quad$ N em boa/nem ruim & 10 & 20,0 \\
Sentimento de estar doente & & \\
$\quad$ Sim & 13 & 26,0 \\
$\quad$ Não & 36 & 72,0 \\
Estágio de soropositividade & & \\
$\quad$ Assintomático & 26 & 52,0 \\
Sintomático & 20 & 40,0 \\
$\quad$ Não sabe & 4 & 8,0 \\
Forma de contágio & & \\
$\quad$ Sexo com homem & 23 & 46,0 \\
Sexo com mulher & 17 & 34,0 \\
$\quad$ Drogas injetáveis & 5 & 10,0 \\
\hline
\end{tabular}


dade inter-entrevistadores foi de 0,934 (IC 95\% $0,879-0,964)(p<0,001)$. Os resultados dos ICC por domínio do HAT-QOL podem ser observados na Tabela 3.

$\mathrm{N}$ o intuito de avaliar graficamente a concordância ou discrepância entre as somas das respostas dadas pelos pacientes no mesmo dia de entrevista, porém utilizando-se entrevistadores diferentes, os resultados das aplicações do H ATQ OL foram plotados em um diagrama de Bland e Altman (Figura 1). Isto permitiu reconhecer tanto a amplitude da variação entre as respostas como reconhecer a existência de vieses sistemáticos entre as duas entrevistas. 0 valor da média aritmética de variação foi igual a 16,05 (IC 95\% 13,54-15,55) ( $D P=7,83)$.

Da mesma forma, foi realizado esse tipo de avaliação comparando o resultado da primeira entrevista com o resultado do índice no reteste (reprodutibilidade intra-entrevistador), que ob- tevemédia aritmética de variação igual a 0,68 (IC $95 \%-0,88-2,25)(\mathrm{DP}=4,97)$ (Figura 2).

Para a validação, aplicou-se o coeficiente de correlação por postos de Spearman entre as respostas dadasna primeira entrevista do H AT-QOL com as respostas dadas ao questionário WHOQOL-HIV-Bref. A correlação entre os valores desses doisíndices diferentesfoi de 0,824 (IC 95\% $0,696-0,901)(p<0,001)$.

\section{Discussão}

A adaptação de um instrumento se faz necessária quando o mesmo foi elaborado em uma cultura diferente daquela na qual vai ser utilizado ou quando a população a que se destina apresenta peculiaridades querequerem modificação deitens do questionário. Guillemin et al. ${ }^{14}$ apontam quea observação de um elevado rigor metodológico no processo de adaptação de questionários é funda-

Tabela 2. Correlação entre os resultados dos domínios H AT-Q OL com o resultado geral e valores de $\alpha$ Cronbach se o domínio for excluído. São Miguel do Oeste (SC), 2006.

\begin{tabular}{lcc}
\hline \multicolumn{1}{c}{ Domínios HAT-QOL } & $\begin{array}{c}\text { Correlação com } \\
\text { outros domínios* }\end{array}$ & $\begin{array}{c}\alpha \text {-Cronbach se o } \\
\text { domínio for excluído }\end{array}$ \\
\hline Funções gerais & 0,709 & 0,872 \\
Funções sexuais & 0,435 & 0,892 \\
Problemas de comunicação & 0,318 & 0,911 \\
Preocupações com a saúde & 0,821 & 0,864 \\
Preocupações financeiras & 0,650 & 0,867 \\
Aceitação sobre o HIV & 0,737 & 0,882 \\
Satisfação sobre a vida & 0,811 & 0,854 \\
Preocupações com medicamentos & 0,500 & 0,891 \\
Confiança no serviço de saúde & 0,296 & 0,893 \\
\hline
\end{tabular}

${ }^{*}$ Correlação de Pearson questão-resultado geral.

Tabela 3. Resultados da análise de reprodutibilidade intra e inter-entrevistador por domínio do HAT-QOL versão brasileira. São M iguel do Oeste (SC), 2006.

\begin{tabular}{lcccccc}
\hline \multirow{2}{*}{ Domínios HAT-QOL } & \multicolumn{2}{c}{ ICC intra-entrevistador* } & & \multicolumn{2}{c}{ ICC inter-entrevistador } \\
\cline { 2 - 3 } \cline { 6 - 7 } & M édia & IC 95\% & & M édia & IC 95\% \\
\hline Funções gerais & 0,950 & $0,913-0,971$ & & 0,955 & $0,922-0,974$ \\
Funções sexuais & 0,962 & $0,933-0,979$ & & 0,961 & $0,931-0,978$ \\
Problemas de comunicação & 0,966 & $0,941-0,980$ & & 0,966 & $0,942-0,981$ \\
Preocupações com a saúde & 0,955 & $0,923-0,974$ & & 0,978 & $0,963-0,988$ \\
Preocupações financeiras & 0,959 & $0,929-0,976$ & & 0,952 & $0,917-0,972$ \\
Aceitação sobre o HIV & 0,933 & $0,884-0,961$ & & 1,000 & $1,000-1,000$ \\
Satisfação sobre a vida & 0,887 & $0,810-0,934$ & & 0,914 & $0,853-0,950$ \\
Preocupações com medicamentos & 0,932 & $0,882-0,961$ & & 0,961 & $0,931-0,978$ \\
Confiança no serviço de saúde & 0,890 & $0,814-0,936$ & & 0,792 & $0,660-0,877$ \\
\hline
\end{tabular}

${ }^{*} p<0,001$. 


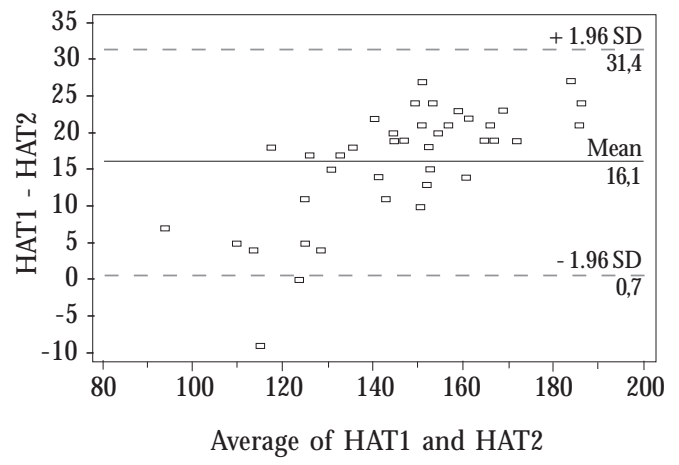

Figura 1. Reprodutibilidade inter-entrevistador do HAT-QOL versão brasileira. Diagrama de Bland e Altman ( $n=40)$ São Miguel do Oeste (SC), 2006.

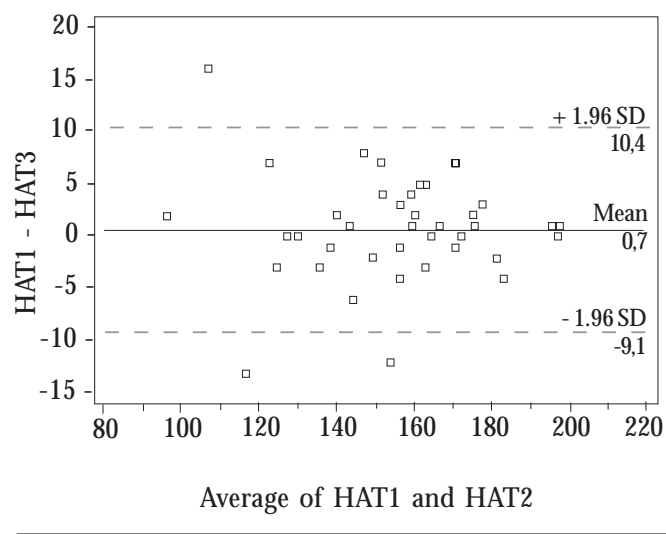

Figura 2. Reprodutibilidade intra-entrevistador do HAT-QOL versão brasileira. Diagrama de Bland e Altman ( $n=41)$. São M iguel do Oeste (SC), 2006.

mental para a validade da comparação entre os resultados e os perfis epidemiológicos obtidos a partir de versões distintas de um mesmo instrumento, empregadas em diferentes localidades e culturas. Logo, a abordagem tradicional, queconsiste geralmente em tradução, retrotradução, comparação da versão com o original, revisão do instrumento por um comitê e teste das propriedades psicométricas do questionário traduzido, senão for realizada com rigor metodológico, pode levar a uma versão que não seja suficiente para que se alcance a equivalência entre as versões original e traduzida do questionário.

No presente estudo, o grau da instrução (62,0\% com ensino fundamental completo, seguidos por $28,0 \%$ com ensino médio completo) pode ter sido um fator que, hipoteticamente, tenha contribuído para que o questionário original fosse mantido em sua estrutura quanto à formulação de questões. Outro fator que possivelmente contribuiu foi o fato de que nenhuma das perguntas referia-se a experiências estranhas à realidade dos pacientes estudados.

A reprodutibilidade, também chamada confiabilidade, é a propriedade demonstrada pelo instrumento dereproduzir resultados semelhantes, quando aplicado em situações semelhantes. N este estudo, os resultados demonstram a existência de confiabilidade adequada do questionário nesse grupo de pacientes. Não se observaram diferenças significativas entre as médias dos escores obtidos nos domínios do questionário quando esse foi aplicado deforma repetida. Ademais, os elevados valores encontrados do ICC intra-entrevistador (0,887 para o domínio "satisfação sobre a vida" a 0,966 para o domínio "problemas de comunicação") e do ICC interentrevistador (0,792 para o domínio "confiança no serviço de saúde" a 1,000 para o domínio "aceitação sobre o HIV") corroboram a afirmação. Entretanto, deve-se ter cautela na interpretação desses dados em função dos intervalos de confiança obtidos. As diferenças al eatórias em relação aos valores médios dos escores de ambos os resultados obtidos nas distintas entrevistas indicam uma menor possibilidade de erros sistemáticos ou vieses (Figuras 1 e 2).

Em relação à consistência interna da versão brasileira do HAT-QOL, observou-se excelente valor, indicado pelo $\alpha$-Cronbach de 0,893. Entretanto, no estudo de correlação dos resultados dos domínios com o resultado geral, observaram-se valores moderados para os domínios "funções sexuais" (ICC =0,435), "preocupações com medicamentos" (ICC $=0,500)$ e baixo no domínio "confiança nos serviços de saúde" (ICC $=0,296)$. Tais resultados são similares aos valores relatados por $\mathrm{H}$ olmes e Shea ${ }^{6}$, em que os mesmos domínios apontaram valores de correlação semelhantes, significando, hipoteticamente, os mesmos problemas encontrados na versão de original. $O$ estudo dos valores de $\alpha$-Cronbach, se cada um dos domínios fosse excluído, mostrou valores bastante altos e próximos entre si (de 0,854 para o domínio "satisfação sobre a vida" a 0,911 para "problemas de comunicação").

A validação é o processo pelo qual se busca demonstrar a capacidade de um instrumento de medir o que se propõe a medir. No presente estudo, correlacionou-se os escores obtidos nas respostas das perguntas do HAT-QOL com as respostas dadas ao questionário WHOQOL-HIV- 
Bref, obtendo-se um alto valor para o coeficiente de correlação por postos de Spearman $(0,824)$. Assim, tendo como precaução a observação do interval o deconfiança obtido, além da necessidade demais estudos para demonstrarem aspectos relevantes à validade do instrumento em sua versão brasileira, os resultados da presente pesquisa sugerem que o HAT-QOL é um instrumento válido para avaliar a QV em pacientes com HIV/aids.

\section{Consideraçõesfinais}

É importante observar que este estudo contou com número limitado de pacientes, tendo sido realizado em uma única instituição, restringindo o universo estudado. Estudos em outras regiões do país e com realidades distintas são importantes antes da utilização plena do questionário na prática clínica.

\section{Referências}

1. Organização Pan-Americana da Saúde. 128a Sessão do Comitê Executivo. Washington, D.C.: OPAS; 2001.

2. Santos ECM. Qualidade de vida de portadores do vírus da imunodeficiência humana [dissertação]. São Paulo (SP): Faculdade de Saúde Pública, Universidade de São Paulo; 2003.

3. The WHOQOL Group. The World Health Organization of Life assessment (WHOQOL): position paper from the World Health Organization. Soc Sci M ed 1995; 41:1403-1409.

4. Fleck M, Fachel O, Leal OF, Louzada S, Xavier M, Chachamovich E, Vieira G, Santos L, Pinzon V. Aplicação da versão em português do instrumento abreviado de avaliação de qualidade de vida "WHOQOL-bref". Rev. Saude Publica 2000; 34:178-183.

5. Ciconelli RM, Ferraz MB, Santos W, M einao I, Quaresma MR. Tradução para o português e validação do questionário genérico de avaliação da qualidade de vida SF-36 (Brasil SF-36). Rev Bras Reumatol 1999; 3:143-150.

6. Holmes WC, Shea JA. Two approaches to measuring quality of life in the HIV/AIDS populations: HAT-QOL and MOS-HIV. Qual Life Res 1999; 8: 515-527.

7. The WHOQOL-HIV Group. Preliminary development of the World Health Organization's Quality of Life HIV instrument (WHOQOL-HIV); analysis pilot version. Soc Sci M ed 2003; 57:1259-1275.

8. Organização Mundial da Saúde. Departamento de Saúde M ental e Dependência Química. WHOQOLHIV BREF. Avaliação de Qualidade de Vida. Genebra: OM S; 2002.
Pode-se concluir que uma versão em português do questionário H AT-QOL foi obtidaa partir detradução eadaptação cultural realizada conformediretrizesinternacionalmenterecomendadas. 0 questionário em sua versão brasileira é prático, de fácil aplicação eapresentou reprodutibilidadeevalidade satisfatórias. Os resultados obtidos suge rem que pode ser usado para a avaliação da qualidade de vida em pacientes com HIV/aids.

\section{Colaboradores}

AM M Moser e J Traebert participaram da concepção, delineamento, análiseeinterpretação dos dados e da redação do artigo.

9. Beaton DE, Bombardier C, Guillemin F, Ferra MB Guidelines for the process of cross-cultural adaptation of self-report measures. Spine 2000; 2: 31863191

10. Wild D, Grove A, Martin M, Eremenco S, M celroy S, Verjee-Lorenz A, Erikson P, ISPOR Task Force for Translation and Cultural Adaptation. Principles of good practice for the translation and cultural adaptation process for patient-reported outcomes (PRO) measures: report of the ISPOR task force for translation and cultural adaptation. Value $\mathrm{Health}$ 2005; 8:94-104.

11. Steiner D, Normann G. Health measurement scales: A practical guide to their development and use. $2^{\text {nd }} \mathrm{ed}$. Oxford: Oxford Medical; 1995.

12. Bland JM, Altman DG. Statistical methods for assessing agreement between two methods of clinical measurement. Lancet 1986; 1:307-310.

13. Siegel S. Estatística não-paramétrica para as ciências do comportamento. São Paulo: M cGraw-Hill; 1975.

14. Guillemin F, Bombardier C, Beaton DE. Cross-cultural adaptation of health-related quality of life measures: literature review and proposed guidelines. J Clin Epidemiol 2003; 46:1417-1432.

Artigo apresentado em 25/03/2008

Aprovado em 31/10/2008

Versão final apresentada em 19/12/2008 